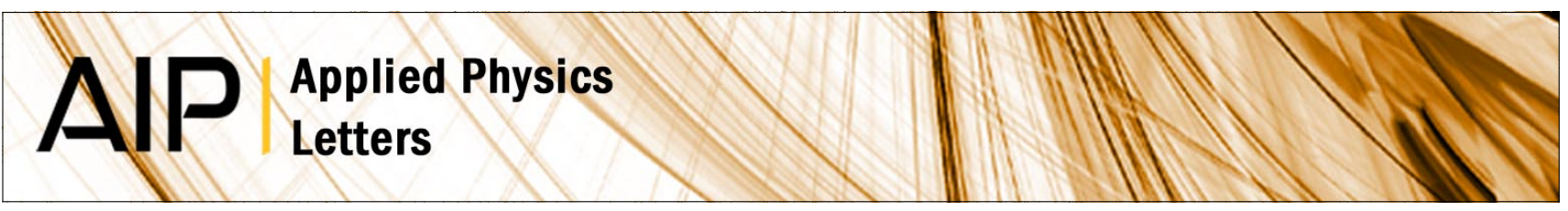

\title{
Sub-bandgap spectral photo-response analysis of Ti supersaturated Si
}

E. García-Hemme, R. García-Hernansanz, J. Olea, D. Pastor, A. del Prado et al.

Citation: Appl. Phys. Lett. 101, 192101 (2012); doi: 10.1063/1.4766171

View online: http://dx.doi.org/10.1063/1.4766171

View Table of Contents: http://apl.aip.org/resource/1/APPLAB/v101/i19

Published by the American Institute of Physics.

\section{Related Articles}

Relevance of intra- and inter-subband scattering on the absorption in heterostructures Appl. Phys. Lett. 101, 191104 (2012)

Microstructural evolution of thin film vanadium oxide prepared by pulsed-direct current magnetron sputtering J. Appl. Phys. 112, 093504 (2012)

Ion implantation induced modification of optical properties in single-crystal diamond studied by coherent acoustic phonon spectroscopy

Appl. Phys. Lett. 101, 181904 (2012)

Making a continuous metal film transparent via scattering cancellations

Appl. Phys. Lett. 101, 181110 (2012)

Three-dimensionally isotropic negative refractive index assisted by two-photon resonance via quantum coherence

Appl. Phys. Lett. 101, 181102 (2012)

\section{Additional information on Appl. Phys. Lett.}

Journal Homepage: http://apl.aip.org/

Journal Information: http://apl.aip.org/about/about_the_journal

Top downloads: http://apl.aip.org/features/most_downloaded

Information for Authors: http://apl.aip.org/authors

\section{ADVERTISEMENT}
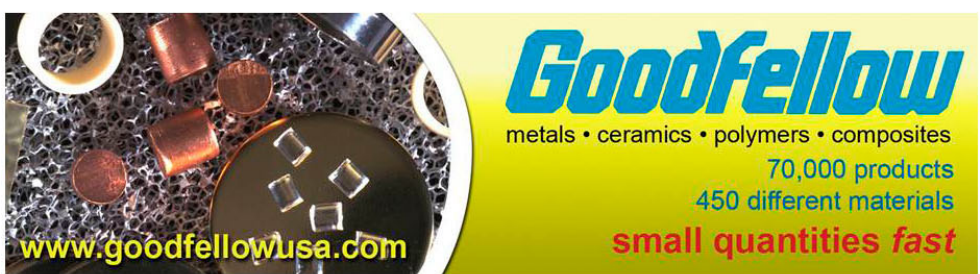


\title{
Sub-bandgap spectral photo-response analysis of Ti supersaturated $\mathbf{S i}$
}

\author{
E. García-Hemme, ${ }^{1,2}$ R. García-Hernansanz,, ${ }^{1,2}$ J. Olea, ${ }^{2,3}$ D. Pastor, ${ }^{1,2,3}$ A. del Prado, ${ }^{1,2}$ \\ I. Mártil, ${ }^{1,2}$ and G. Gónzalez-Díaz ${ }^{1,2}$ \\ ${ }^{1}$ Departamento de Física Aplicada III (Electricidad y Electrónica), Universidad Complutense de Madrid, \\ 28040 Madrid, Spain \\ ${ }^{2}$ CEI Campus Moncloa, UCM-UPM, 28040 Madrid, Spain \\ ${ }^{3}$ Instituto de Energía Solar, E.T.S.I. de Telecomunicación, Universidad Politécnica de Madrid, 28040 Madrid, \\ Spain
}

(Received 24 July 2012; accepted 22 October 2012; published online 6 November 2012)

\begin{abstract}
We have analyzed the increase of the sheet conductance $\left(\Delta G_{\square}\right)$ under spectral illumination in high dose $\mathrm{Ti}$ implanted $\mathrm{Si}$ samples subsequently processed by pulsed-laser melting. Samples with $\mathrm{Ti}$ concentration clearly above the insulator-metal transition limit show a remarkably high $\Delta G_{\square}$, even higher than that measured in a silicon reference sample. This increase in the $\Delta G_{\square}$ magnitude is contrary to the classic understanding of recombination centers action and supports the lifetime recovery predicted for concentrations of deep levels above the insulator-metal transition. (C) 2012 American Institute of Physics. [http://dx.doi.org/10.1063/1.4766171]
\end{abstract}

The infrared range of the electromagnetic spectrum has always been of great attention for the increasing number of practical applications. ${ }^{1}$ Specifically for $\mathrm{Si}$, this response is weak $^{2}$ because of its bandgap energy of $1.12 \mathrm{eV}$. However, recent studies based on $\mathrm{Si}$ highly saturated with chalcogens ${ }^{3,4}$ and $\mathrm{Ti}^{5}$ have shown a wide range of unusual electrical and optical properties, namely, a strong sub bandgap optical absorption and an increase in the infrared photoresponse. One of the hypotheses to explain these properties is the formation of an impurity band of allowed states within the bandgap of the host semiconductor once the impurity concentration has overcome the so-called insulator-metal transition. This impurity band would allow optical transitions for photon energies below the bandgap which, together with a recovery of the carrier lifetime, would lead to an increase in the photo-response at these photon energies. In this context, these materials are being studied for potential photovoltaic applications since the exploitation of the infrared region of the solar spectrum in $\mathrm{Si}$ based solar cells is a goal to achieve by the photovoltaic researchers community. The concept of a solar cell based in this impurity band material is known as intermediate band (IB) solar cell and would lead to a remarkably increase in the solar cell efficiency. ${ }^{6}$

Different approaches to obtain an IB material have been reported, such as quantum dots and highly mismatched alloys. ${ }^{7,8}$ Another method is the introduction of deep centers at a very high concentration in the semiconductor. Typically these impurities act as non-radiative recombination (NRR) centers, reducing drastically the carrier lifetime. ${ }^{9}$ However, at very high impurity concentrations, the electron wave functions would overlap producing a delocalization of the states associated to the impurities. Then, a localization-delocalization transition similar to the well-known insulator-metal transition described by Mott is achieved. ${ }^{10}$ The concentration that determines the insulator-metal transition limit (Mott limit) has been theoretically calculated to be approximately $6 \times 10^{19} \mathrm{~cm}^{-3} \cdot 11$ Once this limit is achieved, the reduction of the NRR is possible and an increase in the charge carrier lifetime may take place as it is explained by the configuration diagram theory in Ref. 11 .
Ti would be an excellent candidate to form an impurity IB in $\mathrm{Si}$ as it has been shown in a previous theoretical study. $^{12}$ The combination of non-equilibrium thermodynamic techniques such as ion implantation and pulsed laser melting (PLM), is a powerful tool to fabricate these IB materials. ${ }^{5}$ These techniques allow a high solute trapping and can incorporate the implanted impurities well above the solid solubility limit of $\mathrm{Ti}$ in $\mathrm{Si}$ and even over the Mott limit., ${ }^{43}$

The crystalline quality of the Ti implanted and subsequently PLM Si layers has been reported elsewhere. ${ }^{13} \mathrm{~A}$ complete electrical characterization of this material has been also performed showing unusual electrical transport properties due to the bilayer structure Ti implanted layer/n-Si substrate. These electrical transport characteristics have been well explained using an analytical model ${ }^{14}$ and a numerical simulation program ${ }^{15}$ assuming in both cases the formation of an IB in the implanted layer of the Si sample. In relation with the optical properties, remarkably high sub-bandgap absorption has been also reported recently ${ }^{5}$ being the measured values for the absorption coefficient in the implanted layer in the order of $10^{3}-10^{4} \mathrm{~cm}^{-1}$.

Here, we will analyze the bilayer photoresponse for sub bandgap energies relating its specific features to the Ti concentration in the implanted layer.

Samples $1 \times 1 \mathrm{~cm}^{2}$ in size of n-type $\mathrm{Si}(100)$ with a thickness of $525 \mu \mathrm{m}\left(\rho \approx 3600 \Omega \mathrm{cm} ; \mu \approx 1500 \mathrm{~cm}^{2} \mathrm{~V}^{-1} \mathrm{~s}^{-1}\right.$; $\mathrm{n} \approx 10^{12} \mathrm{~cm}^{-3}$ at room temperature) were implanted in a refurbished VARIAN CF3000 Ion Implanter at $32 \mathrm{keV}$ with different Ti doses $\left(10^{13}, 10^{14}, 10^{15}\right.$, and $\left.10^{16} \mathrm{~cm}^{-2}\right)$ using a $7^{\circ}$ tilt angle. After implantation, all the samples were PLM processed at $1.4 \mathrm{~J} / \mathrm{cm}^{2}$ with a $\mathrm{KrF}$ excimer laser $(248 \mathrm{~nm})$ at J.P. Sercel Associates, Inc. (New Hampshire, USA).

To analyze the $\mathrm{Ti}$ depth profile, time-of-flight secondary ion mass spectrometry (ToF-SIMS) measurements were carried out in a ToF-SIMS IV model manufactured by ION-TOF, with a $25 \mathrm{keV}$ pulsed $\mathrm{Bi}^{3+}$ beam at $45^{\circ}$ incidence. A $10 \mathrm{keV}$ voltage was used to extract the secondary ions generated and their time of flight from the sample to the detector was measured with a reflection mass spectrometer. The structural characterization of 


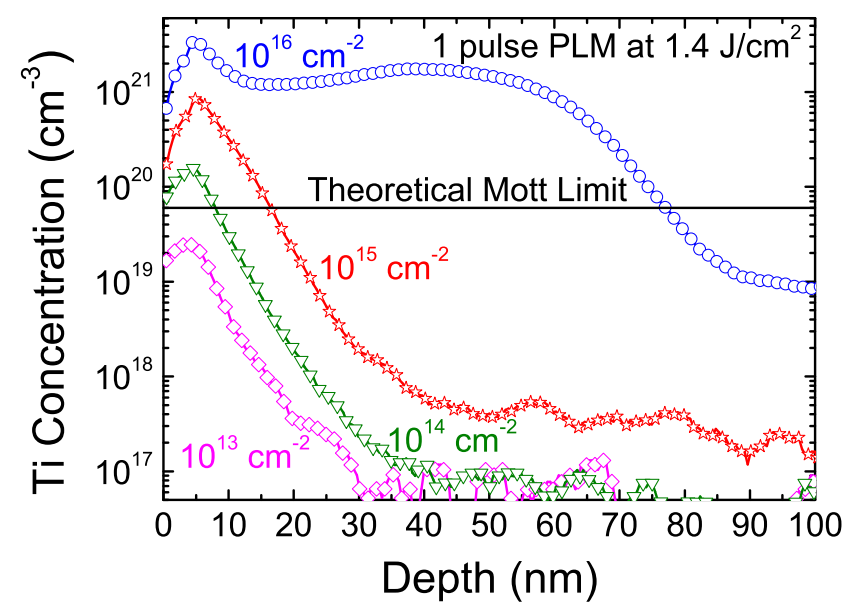

FIG. 1. ToF-SIMS profiles of Ti implanted Si samples with doses of $10^{13}$, $10^{14}, 10^{15}$, and $10^{16} \mathrm{~cm}^{-2}$ and subsequently PLM at $1.4 \mathrm{~J} / \mathrm{cm}^{2}$. The three implanted samples with the highest doses have Ti concentrations above the theoretical Mott limit.

similar samples was carried out by transmission electron microscopy (TEM) and electron diffraction (ED) patterns and is reported elsewhere. ${ }^{13}$

Finally, the sample spectral photo-conductance for energies below the bandgap was analyzed using the van der Pauw set up. For that, four small Al triangular contacts were e-beam evaporated in the sample corners, on top of the Ti implanted layer. A fixed current was injected through two contacts and the AC voltage generated over the opposite two contacts was measured while a monochromatic chopped light impinges the samples. A TMc300 Bentham monochromator with a halogen lamp source was used and the intensity of the light was calibrated with a Bentham pyrometric detector. Samples were placed in a homemade liquid-nitrogen cryostat with a $\mathrm{ZnSe}$ window. Measurements were carried out at $90 \mathrm{~K}$ to reduce the thermal noise and a vacuum pump was used to avoid moisture condensation at low temperature. DC currents in the order of $1 \mathrm{~mA}$ or lower were used for the van der Pauw measurement to avoid self-heating effects. Measurements were carried out with a SR830 digital signal processing lock-in amplifier manufactured by Stanford Research Systems (California). For all of the measurements, the light from the source was mechanically chopped at $23.5 \mathrm{~Hz}$. This frequency is low enough to allow the sample response to reach steady states both in illumination and in darkness, as we have checked out by monitoring the transient of the response.

Figure 1 shows the $\mathrm{Ti}$ concentration depth profiles obtained by ToF-SIMS for samples implanted with the four

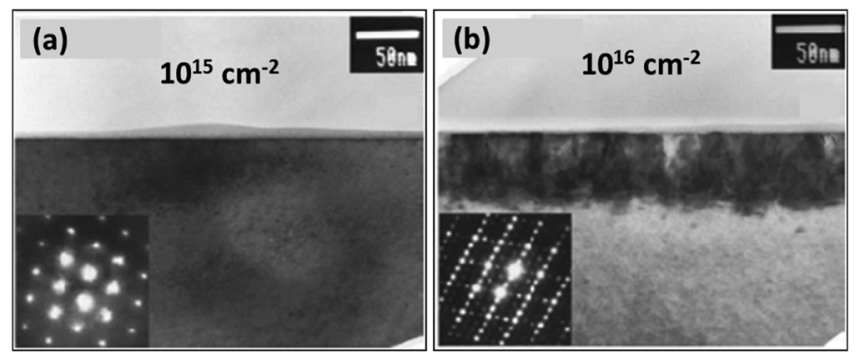

FIG. 2. Cross sectional TEM images and ED images of samples implanted with the doses of $10^{15} \mathrm{~cm}^{-2}$ (a) and $10^{16} \mathrm{~cm}^{-2}$ (b) and subsequently processed by PLM. different Ti doses and subsequently PLM processed. The theoretical Mott limit is plotted as a reference. For the sample implanted with the lowest dose $\left(10^{13} \mathrm{~cm}^{-2}\right)$, the Mott limit concentration is not reached. However, for the samples implanted at higher doses, a Ti concentration over the theoretical Mott limit is reached in a layer with an approximate thickness of 10,20 , and $80 \mathrm{~nm}$ for the $10^{14}, 10^{15}$, and $10^{16} \mathrm{~cm}^{-2}$ doses, respectively. Additionally, higher Ti concentrations are obtained as the implanted dose is increased. Theoretically, the delocalization transition point could have been surpassed for some of the samples and we could expect a reduction of the NRR at the Ti implanted layer. ${ }^{11}$

Figure 2 shows TEM and ED images of similar samples implanted with the dose of $10^{15} \mathrm{~cm}^{-2}$ (a) and $10^{16} \mathrm{~cm}^{-2}$ (b) and subsequently processed by PLM at $0.8 \mathrm{Jcm}^{-2} \cdot 13$ An excellent crystalline structure after the PLM process is obtained for the $10^{15} \mathrm{~cm}^{-2}$ doses, as shown in Fig. 2(a). A single crystalline layer is obtained in spite of the high Ti concentration. In addition, no differences between the ED pattern obtained for the processed layer and the ED of a silicon reference substrate are observed. These results point to a very high crystal lattice reconstruction. For the samples implanted at $10^{13}$ and $10^{14} \mathrm{~cm}^{-2}$ doses, we have observed clear evidences of a high crystal quality by means of Raman spectroscopy measurements. On the other hand, for the $10^{16} \mathrm{~cm}^{-2}$ implanted sample, a defective layer on top of a single crystalline silicon substrate is observed. Regarding the ED patterns, we observe a bright spot pattern that corresponds to the [111] zone axes of the silicon. However, intermediate spots appear between the main dots which have been associated with the highly defective lattice reconstruction. ${ }^{16}$

Figure 3 shows the results of the sheet conductance increase $\left(\Delta G_{\square}\right)$ magnitude normalized to the incident light power $\mathrm{I}_{0}$, as a function of the energy of the incident photons. The $\Delta G_{\square}$ magnitude is presented for the samples processed with all the different implanted doses and for a reference $\mathrm{Si}$ substrate. Due to the high noise, $\Delta G_{\square}$ is not represented below $0.5,0.6$, and $0.85 \mathrm{eV}$ for the $\mathrm{Si}$ reference substrate, the $10^{14} \mathrm{~cm}^{-2}$ and the $10^{13} \mathrm{~cm}^{-2}$ implanted samples,

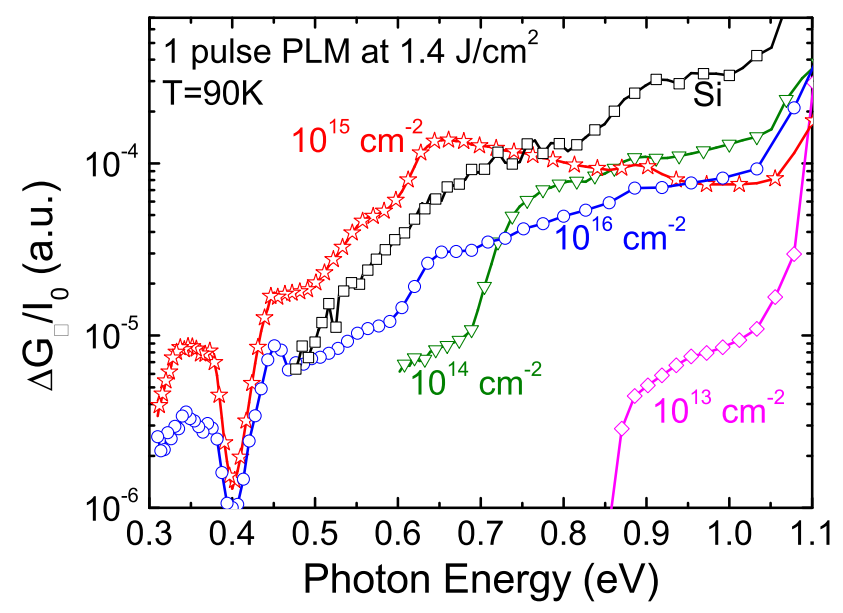

FIG. 3. Variation of the sheet conductance spectral response normalized to the impinging light power as a function of the incident photon energy for the Si unimplanted reference sample, and for the four implanted samples with $10^{13}, 10^{14}, 10^{15}$, and $10^{16} \mathrm{~cm}^{-2}$ doses and subsequently PLM at $1.4 \mathrm{~J} / \mathrm{cm}^{2}$. Measurements were carried out at $90 \mathrm{~K}$. 
respectively. For energies higher than $1 \mathrm{eV}$, the absorption edge of the Si bandgap is clearly observed for all the samples. A noticeable $\Delta G_{\square}$ magnitude is observed for the $\mathrm{Si}$ substrate even for energies below the bandgap. It must be noted that the doping of the substrate is very low, leading to high carrier lifetime, which is the responsible of the high $\Delta G_{\square}$ measured values.

The sheet resistance of a uniform sample like the reference substrate in a darkness state $R_{\square d}$ (or its inverse, the sheet conductance $G_{\square d}$ ) was defined by van der Pauw as ${ }^{17}$

$$
R_{\square d}=\frac{1}{G_{\square d}}=\frac{\pi}{\ln 2} \frac{V_{d}}{I},
$$

where $I$ is the fixed current intensity injected into the sample through two adjacent contacts, and $V_{d}$ is the voltage drop in a darkness state over the opposite two contacts. An analogous expression would be obtained for the illumination state replacing the subscript $d$ by $i$. This macroscopic magnitude is related with the fundamental electrical transport parameters for a n-type semiconductor

$$
R_{\square d}=\frac{1}{G_{\square d}}=\frac{1}{q \mu n_{d} t},
$$

where $q$ is the electron charge, $\mu$ is the carrier mobility, $n_{d}$ is the carrier concentration in darkness, and $t$ is the sample thickness.

Under illumination, optical transitions take place, increasing the charge carrier concentration. In an illuminated steady state, the carrier concentration can be defined as: $n_{i}=n_{d}+\Delta n$, where $\Delta n$ is the increase in the carrier concentration with respect to the darkness situation. This excess of carriers, $\Delta n$, leads to a variation in the sheet conductance and therefore a variation of the voltage drop measured in the van der Pauw set up: $V_{i}=V_{d}+\Delta V$. The difference of the voltage drop corresponding to darkness and illumination states $\Delta V$ is the voltage amplitude measured using the lockin amplifier set up. Considering Eq. (1), for darkness and illumination, and taking into account that $\Delta V \ll V_{d}$, the measured $\Delta V$ is related to the increase of the sheet conductance by

$$
\Delta V=-V_{d} \frac{\Delta G_{\square}}{G_{d}},
$$

where $\Delta G_{\square}$ is the increase of the sheet conductance due to the illumination which is related to $\Delta n$ by its definition, according to Eq. (2): $\Delta G_{\square}=q \mu \Delta n t$. The sign in Eq. (3) just indicates a $180^{\circ}$ phase difference.

Considering that the chopping frequency is low enough to obtain an illuminated steady state, the charge carrier generation rate has to be equal to the recombination rate. The generation rate can be defined as: $g=\alpha \eta I_{0} t$, where $\alpha$ is the optical absorption coefficient, $\eta$ is the quantum yield, and $I_{0}$ is the incident power of light (impinging photons that reach the sample per second and $\mathrm{cm}^{2}$ ). To express the generation rate in this way, we have assumed that $\alpha t \ll 1$ which is a good approximation both for the substrate and for the implanted layer. In the former case, $\alpha$ is very low although unknown for energies well below the bandgap (which is our interest area) and in the second one because the implanted thickness is lower than $10^{-5} \mathrm{~cm}$ as it is shown in Fig. 1 and the $\alpha$ coefficient is lower than $10^{4} \mathrm{~cm}^{-1}$. The recombination rate can be defined as: $r=\Delta n / \tau$, where $\tau$ is the charge carrier lifetime. Then, assuming steady state conditions $(g=r)$ and using the definition of $\Delta G_{\square}$, we can relate it with the charge carrier lifetime in the reference substrate as follows:

$$
\frac{\Delta G_{\square}}{I_{0}}=q \mu t \eta \alpha \tau
$$

The experimental variation of the $\Delta G_{\square} / I_{0}$ magnitude and its relation with the lifetime of carriers will be assessed in the next paragraphs.

The sample implanted with the lowest dose $\left(10^{13} \mathrm{~cm}^{-2}\right)$ presents values of $\Delta G_{\square}$ two orders of magnitude lower than the corresponding $\Delta G_{\square}$ values obtained for the silicon reference sample and its photo-response decreases drastically for irradiation energies below $0.85 \mathrm{eV}$. Dark DC measurements of this sample show the same sheet conductance as the substrate from room temperature to $90 \mathrm{~K}$. At $90 \mathrm{~K}$, the sheet conductance showed a value of $10.87 \times 10^{-5} \mathrm{~S}$. The coincidence on these measurements implies that we are dealing with two layers in parallel (implanted layer/substrate) with no limitation for the electrical conduction between them and that the sheet conductance of the implanted layer is much lower than the sheet conductance of the substrate (It must be noted that the thickness and therefore the sheet conductance of the substrate layer is unaffected by the formation of the very thin implanted layer).

Extending Eq. (3) to the case of a parallel bilayer, we obtain

$$
\Delta V_{b}=-V_{b d} \frac{\Delta G_{\square l}+\Delta G_{\square s}}{G_{\square l d}+G_{\square s d}}=-V_{b d} \frac{\mu_{l} t_{l} \Delta n_{l}+\mu_{s} t_{s} \Delta n_{s}}{\mu_{l} t_{l} n_{l}+\mu_{s} t_{s} n_{s}},
$$

where $\Delta V_{b}$ is the voltage generated in the bilayer and the subscript $l$ is relative to the implanted layer and $s$ for the substrate. As explained before, for the $10^{13} \mathrm{~cm}^{-2}$ sample, the value of $G_{\square l d}$ is much lower than $G_{\square s d}$. It must be expected also that $\mu_{l} t_{l} \Delta n_{l}$ will be smaller than $\mu_{s} t_{s} \Delta n_{s}$ because the presence of greats amounts of Ti does not allow the presence of surplus carriers on the implanted layer and also because $t_{l} \ll t_{s}$ and $\mu_{l}<\mu_{s}$. Consequently, the observed photovoltage should be the same than for the substrate reference, i.e., $\Delta V_{b}=-V_{s d} \frac{\Delta G_{\square s}}{G_{\square d d}}=-V_{s d} \frac{\Delta n_{s}}{n_{s}}$. As the experimental measurements of the sample implanted at $10^{13} \mathrm{~cm}^{-2}$ shows $\Delta V_{b}$ values orders of magnitude lower than the ones obtained for the reference substrate, we have to conclude that the presence of a high Ti concentration in the upper layer also modified the photocarriers density at the substrate $\Delta n_{s}$. As stated before, the high resistivity substrate has a very high mean free path, higher than the substrate thickness, producing a strong interaction with the wafer surfaces. The interface between the implanted layer and the substrate is characterized by a high recombination velocity due to the high concentration of deep centers. Therefore, there is a great probability for the annihilations of the photocarriers which interacts with it. In fact, it is well establish that in this situation the lifetime can be 
written as $1 / \tau_{\text {eff }}=1 / \tau_{s}+S$, where $\tau_{\text {eff }}$ is the effective lifetime, $\tau_{s}$ is the lifetime in the reference substrate, and $S$ is the recombination velocity at the implanted layer/substrate interface. If $S$ has a high value which is in agreement with the high Ti concentration, it should be expected an important decrease of the substrate photoconductivity, which leads to the low measured values of the bilayer photoconductance $\Delta G_{\square b}$ for this sample as can be seen in Fig. 3.

The sample implanted with $10^{14} \mathrm{~cm}^{-2}$ dose (dark sheet conductance of $10.87 \times 10^{-5} \mathrm{~S}$ ) shows higher photoconductivity, which is not congruent with the previous explanation as we have higher Ti concentration, except if we accept that the recombination velocity at the implanted layer/substrate interface is becoming lower. According to Fig. 1, this sample has $\mathrm{Ti}$ concentration over the Mott limit but in a very reduced thickness. Therefore, the insulator-metal transition takes place and then a reduction of the recombination velocity is expected at least in this thinner layer.

For sample implanted with $10^{15} \mathrm{~cm}^{-2}$ dose (dark sheet conductance of $10.03 \times 10^{-5} \mathrm{~S}$ ), we can observe in Fig. 3 that the photoconductivity could now be detected for a wider energy range going down to $0.3 \mathrm{eV}$ and is higher for the bilayer than for the substrate for some energy range. As it was shown in previous published papers related with electrical transport measurements, ${ }^{14}$ the formation of the IB is well established for this dose. In this sense, the previously analyzed $10^{14} \mathrm{~cm}^{-2}$ sample could be considered a transition sample between a Ti rich sample and a sample where the IB is fully formed. It is not reasonable to assume that for the sample implanted at the dose of $10^{15} \mathrm{~cm}^{-2}$ the interface recombination velocity $S$ is reduced to 0 in spite of the IB formation but it is surely much lower that for the samples implanted at $10^{13}$ or $10^{14} \mathrm{~cm}^{-2}$ doses. The increase of the photoconductivity over the substrate values could be explained having in mind Eq. (5). First, there is a recovery of the lifetime that will increase $\Delta n_{s}$ drastically to values comparable but anyway lower than in the case of the substrate alone. Second, an increase of the photocarriers in the implanted layer could made $\mu_{l} t_{l} \Delta n_{l}$ comparable or higher than $\mu_{s} t_{s} \Delta n_{s}$ and consequently an increase in $\Delta G_{\square b}$ over the substrate photoconductance could be observed.

For the $10^{15} \mathrm{~cm}^{-2}$ sample, the condition $G_{\square l d} \ll G_{\square s d}$ still holds (since $t_{l} \ll t_{s}$ ) and the voltage increase could be written as

$$
\Delta V_{b}=V_{d b} \frac{1}{n_{s}}\left(\frac{\mu_{l} t_{l} \Delta n_{l}}{\mu_{s} t_{s}}+\Delta n_{s}\right)
$$

which could be larger than the substrate value.

We have to note that $\Delta n_{l}$ could increase due to two facts: the increase of the light absorption in the implanted layer and also the increase of the effective lifetime due to the formation of the IB. Anyway, the increase of $\Delta n_{l}$ has to be very important to compensate the diminishing effect of $\frac{\mu_{l} t_{l}}{\mu_{s} t_{s}}$ which might be in the order of $10^{-3}$. We observe very small differences in the measured transmittance for samples implanted with $10^{14} \mathrm{~cm}^{-2}$ and $10^{15} \mathrm{~cm}^{-2}$ with respect to the transmittance of the Si reference sample. Then, the increase produced in the $\Delta G_{\square}$ magnitude cannot be justified completely by the increase of the absorption coefficient in $\frac{\Delta G_{\square}}{I_{0}}=q \mu t \eta \alpha \tau$.
Therefore, an increase of the lifetime due to the reduction of the non-radiative recombination in the implanted layer/Si substrate interface is necessary to explain the results.

These results are in full agreement with a previous work in which the authors measured an increase of the carrier lifetime on Si wafers supersaturated with Ti implanted at $10^{15}$, $5 \times 10^{15}$, and $10^{16} \mathrm{~cm}^{-2}$ doses $^{18}$ using the quasi-steady-state photoconductance technique. ${ }^{19}$

Finally, the sample implanted with the highest dose $\left(10^{16} \mathrm{~cm}^{-2}\right.$, dark sheet conductance of $\left.68.35 \times 10^{-5} \mathrm{~S}\right)$ presents slightly lower values of $\Delta G_{\square}$ than the sample implanted with the dose of $10^{15} \mathrm{~cm}^{-2}$, even though it contains a higher Ti concentration. We could relate this behavior with the defective lattice reconstruction expected for this sample due to the high implantation dose, whereas the samples implanted with lower doses show a far better lattice reconstruction, as previously discussed concerning Figs. 2(a) and 2(b). ${ }^{13}$ The presence of any kind of defect, like twin boundaries, stacking faults, dislocations, or even the cellular breakdown could have a detrimental effect in terms of a stronger recombination, which would result in a decrease of $\Delta G_{\square}$, as observed.

In conclusion, Ti supersaturated Si layers were obtained by means of two non-equilibrium thermodynamic processes: ion implantation and PLM. Samples were implanted with different Ti concentrations, below and above the theoretical Mott limit. As the Ti concentration increases, an increase of the $\Delta G_{\square}$ spectral response is observed. This $\Delta G_{\square}$ increase has been related to the increase of the carrier life time predicted by the insulator-metal transition theory. ${ }^{11}$ These results could lead to the development of a novel generation of semiconductor devices based in Si with enhanced photoresponse in the infrared range of the spectrum.

Authors would like to acknowledge the CAI de Técnicas Físicas of the Universidad Complutense de Madrid for the ion implantations and metallic evaporations and the Nanotechnology and Surface Analysis Services of the Universidad de Vigo C.A.C.T.I. for ToF-SIMS measurements. This work was partially supported by the Project NUMANCIA II (Grant No. S-2009/ENE/1477) funded by the Comunidad de Madrid. Research by E. García-Hemme was also supported by a PICATA predoctoral fellowship of the Moncloa Campus of International Excellence (UCM-UPM). J. Olea and D. Pastor thanks Professor A. Martí and Professor A. Luque for useful discussions and guidance and acknowledge financial support from the MICINN within the program Juan de la Cierva (JCI-2011-10402 and JCI-2011-11471), under which this research was undertaken.

\footnotetext{
${ }^{1}$ A. Rogalski, Prog. Quantum Electron. 27, 59-210 (2003).

${ }^{2}$ D. J. Lockwood and L. Pavesi, in Silicon Photonics (Springer, 2004), Vol. 94, pp. 1-50.

${ }^{3}$ A. J. Said, D. Recht, J. T. Sullivan, J. M. Warrender, T. Buonassisi, P. D. Persans, and M. J. Aziz, Appl. Phys. Lett. 99, 073503 (2011).

${ }^{4}$ M. Tabbal, T. Kim, D. N. Woolf, B. Shin, and M. J. Aziz, Appl. Phys. A: Mater. Sci. Process. 98, 589-594 (2010).

${ }^{5}$ J. Olea, Á. del Prado, D. Pastor, I. Mártil, and G. González-Díaz, J. Appl. Phys. 109, 113541 (2011).

${ }^{6}$ A. Luque and A. Martí, Phys. Rev. Lett. 78, 5014-5017 (1997).

${ }^{7}$ A. Luque, A. Martí, and C. Stanley, Nat. Photonics 6, 146-152 (2012).

${ }^{8}$ N. Ahsan, N. Miyashita, M. M. Islam, K. M. Yu, W. Walukiewicz, and Y. Okada, Appl. Phys. Lett. 100, 172111 (2012).
} 
${ }^{9}$ J. R. Davis, A. Rohatgi, R. H. Hopkins, P. D. Blais, P. Raichoudhury, J. R. McCormick, and H. C. Mollenkopf, IEEE Trans. Electron Devices 27(4), 677-687 (1980).

${ }^{10}$ N. F. Mott, Adv. Phys. 21(94), 785-823 (1972).

${ }^{11}$ A. Luque, A. Martí, E. Antolín, and C. Tablero, Phys. B: Condens. Matter 382(1-2), 320-327 (2006).

${ }^{12}$ K. Sánchez, I. Aguilera, P. Palacios, and P. Wahnon, Phys. Rev. B 79, 165203 (2009).

${ }^{13}$ J. Olea, M. Toledano-Luque, D. Pastor, E. San-Andrés, I. Mártil, and G. González-Díaz, J. Appl. Phys. 107, 103524 (2010).
${ }^{14}$ J. Olea, G. González-Díaz, D. Pastor, I. Mártil, A. Martí, E. Antolín, and A. Luque, J. Appl. Phys. 109, 063718 (2011).

${ }^{15}$ J. Olea, G. González-Díaz, D. Pastor, and I. Mártil, J. Phys. D: Appl. Phys. 42, 085110 (2009).

${ }^{16}$ D. B. Williams and B. C. Carter, Transmission Electron Microscopy: Difraction (Plenum, New York, USA, 1996).

${ }^{17}$ L. J. van der Pauw, Philips Tech. Rev. 20, 220-224 (1958).

${ }^{18}$ E. Antolín, A. Martí, J. Olea, D. Pastor, G. González-Díaz, I. Mártil, and A. Luque, Appl. Phys. Lett. 94, 042115 (2009).

${ }^{19}$ R. A. Sintón and A. Cuevas, Appl. Phys. Lett. 69, 2510-2512 (1996). 